\title{
Mathematical actions as procedural resources: An example from the separation of variables
}

\author{
Michael C. Wittmann \\ Department of Physics and Astronomy, Maine Center for Research in STEM Education, \\ University of Maine, Orono, Maine 04469, USA \\ Katrina E. Black \\ Department of Physics, Michigan Technical University, Houghton, Michigan 49931, USA
}

(Received 29 September 2014; published 23 September 2015)

\begin{abstract}
[This paper is part of the Focused Collection on Upper Division Physics Courses.] Students learning to separate variables in order to solve a differential equation have multiple ways of correctly doing so. The procedures involved in separation include division or multiplication after properly grouping terms in an equation, moving terms (again, at times grouped) from one location on the page to another, or simply carrying out separation as a single act without showing any steps. We describe student use of these procedures in terms of Hammer's resources, showing that each of the previously listed procedures is its own "piece" of a larger problem solving activity. Our data come from group examinations of students separating variables while solving an air resistance problem in an intermediate mechanics class. Through detailed analysis of four groups of students, we motivate that the mathematical procedures are resources and show the issues that students must resolve in order to successfully separate variables. We use this analysis to suggest ways in which new resources (such as separation) come to be.
\end{abstract}

DOI: 10.1103/PhysRevSTPER.11.020114

PACS numbers: 01.40.Ha

\section{INTRODUCTION}

As part of a project to describe mathematical problem solving in physics from a resources perspective [1-6], we have looked at students' understanding of variables [7], understanding of integration constants [8], the use of multiple methods for finding unknown integration constants $[9,10]$, and students' choice of epistemic games [11] when carrying out integration. While carrying out these studies, we regularly observed students struggling with algebra in some contexts and not in others. There is an extensive literature on the teaching and learning of algebra (as summarized in Refs. $[12,13])$, but little work has been done in physics. One area in which students struggle with algebraic manipulation is the separation of variables, used, for example, to isolate terms in a first order differential equation so that one can integrate the two sides of an equality independently. We have found that students who are otherwise strong in their algebraic skills (as observed in classroom activities or on examinations) have difficulties when separating variables. In this paper, we use the discussion of students resolving their difficulties to extend our understanding of student reasoning in a physics class.

We model reasoning in terms of a theoretical framework focused explicitly on variability of students' reasoning in

Published by the American Physical Society under the terms of the Creative Commons Attribution 3.0 License. Further distribution of this work must maintain attribution to the author(s) and the published article's title, journal citation, and DOI. problem solving: Hammer's resources [1]. This framework has been valuable in understanding conceptual [3-6] and epistemological [14-16] reasoning as well as the transfer of knowledge [2]. We extend the framework to include mathematical actions, which we call "procedural resources" [9]. We show that mathematical actions meet more than one of the definitions of resources (two of which are discussed below). This enriches our understanding of the use of mathematics in physics, suggesting both instructional and analytical tools to expand our knowledge of learning in physics.

To make our case, we look at just one step in a long problem in which students needed to describe the motion of a thrown object. As part of the problem, students are required to separate variables. The task is a relatively simple one, as long as one understands that the derivative (in this case, $d v / d t$ ) can be treated as a fraction. We do not observe students having problems with that aspect of the problem. Instead, they struggle with how to group terms appropriately in order to create an equation with variables isolated on each side of the equation. (Other aspects of our work investigate what happens after this point, as students begin to integrate [9-11].)

We begin this paper with a discussion of the resources framework, including a brief discussion of local coherence and compilation of resources. This is followed by a description of our research setting and analysis methods. Next, we present data from four groups discussing the separation of variables in an air resistance problem. Finally, we discuss our results and their meaning within the 
resources framework and their implications for instruction and curriculum design. Our central point is that the mathematical actions discussed in this paper can be thought of as procedural resources.

\section{MODELING STUDENTS' IDEAS USING THE RESOURCES FRAMEWORK}

The reasoning resources framework proposed by Hammer [1] and extended by Hammer, Redish, and others $[2-6,17,18]$ was introduced as a kind of "knowledge in pieces" approach to thinking [18-24] with a specific emphasis on problem solving in physics. We think of knowledge pieces as schemalike units of thinking which are activated in a given setting to reason about a given topic. Most of the "pieces" discussed in the physics education research literature (see, for example, Refs. [5,21,23]) describe these as basic, "primitive" ideas which are developed early in life and used in later life to make sense of complicated new situations.

Several kinds of small-scale conceptual knowledge have been described in the literature, including "agents" [18], "facets of knowledge" [20], "phenomenological primitives" (p-prims) [21], "mathematical forms" [22,25], and "intuitive rules" [24]. A discussion of the differences between these different descriptions of knowledge is beyond the scope of this paper. In our work, we have used Hammer's "resources" [1]. Hammer describes them as elements of a problem solving activity that can be of any cognitive scale. We make an analogy to printing a document from a computer. One would need a printer, drivers, and a program from which to print the document. Each of these - the program, the driver, or the printer-is a resource when attempting to print the desired document. They are of different natures (software and hardware) and scales (a driver is a small piece of code in the operating system, specific to the printer; a program is a larger piece of code independent of the operating system, where many programs can print to the printer). They are each necessary in their own way, and solving the problem of printing requires that all be used in the correct order. Finally, they are all useful, though they might be useless until used in conjunction with other resources.

Resources, like most schemalike theories, are helpful in organizing our description of knowledge into "chunks" of information, consistent with what we know about working memory. Working memory is fast but limited [26]; it can hold only a few items at a time, and those only for a few seconds (see, for example, Ref. [27]). However, the "items" in working memory may encode considerable structure. Resources, as described above, are one way of describing the at times highly encoded structure of chunks of knowledge. Even very basic knowledge pieces might have considerable substructure. A knowledge piece like dying away, a phenomenological primitive (p-prim) described by DiSessa [21], requires an object, a property of the object, a time-dependent change in the property, and that the change be a gradual lessening. This is clearly not a primitive idea when treated analytically, but dying away is used readily and easily to describe the sound of a rung bell and the motion of an object given a shove across a floor. In this paper, we discuss student reasoning in terms of the procedural resources of problem solving that eventually come to be an easily usable resource. We provide examples of the resources in our data and discuss creation of new resources after.

Before we are able to do so, we must first determine whether the mathematical actions we observe students engaged in can be thought of as procedural resources. We look to the descriptions of resources given in the literature. Sayre and Wittmann [28] describe resources as "individual reusable thoughts" that can exist in two states (activated in a situation or not) and are connected to other thoughts, while having some level of internal structure that is perhaps apparent to the user, but possibly not. We show below that each of these criteria is met by our procedural resources. We also consider Scherr's analysis of the pieces model [17], in which knowledge pieces are mutually independent, context dependent, and indeterminate. We show that these criteria are also met in our analysis of procedural resources.

The resources framework allows for the idea that resources can develop at any time in one's learning career and can be applied usefully in many different settings. Ideas about resource creation include compilation [29] of a locally coherent set of resources [2] and reification of processes into mental objects [30-32]. Our work adds to the discussion of the resources framework by providing examples of students who have not yet compiled the procedural resource of separating, suggesting that there are multiple ways of doing so.

In the discussion, we revisit these points and discuss the advantages of using a resources framework to explain student mathematical problem solving in physics.

\section{RESEARCH DESIGN}

The work described in this paper was part of a larger project to study student use of mathematics in advanced physics courses at a large state university [7-11]. Part of our larger project was to understand student use of mathematics in an advanced physics class and develop learning materials to help these students in their learning. Thus, our attention was focused on student use of mathematics, their difficulties and strengths, and the ways in which these were manifested in group learning activities.

In this section, we describe the classroom setting, including a description of the student population studied. We describe the problem we studied and its possible solutions. We describe how the sample of students studied was chosen, and end with a discussion of constraints on our data collection and its effects on our analysis. 


\section{A. Classroom setting}

The study took place in a sophomore-level mechanics course in which students are required to have a course in differential equations as a corequisite. Class size was between 12 and 20 students. The framework of the mechanics course was primarily Newtonian, with only brief discussions of Lagrangian mechanics and the variational principle. The topic of first order linear differential equations typically came in the first few weeks of the course.

Students in the course used the Intermediate Mechanics Tutorials [33-35] in about a third of their classes. In these activities, students work to combine their conceptual and mathematical knowledge of mechanics using guided questions. These small group learning activities are based on the Tutorials in Introductory Physics [36] as well as the Activity-Based Tutorials [37,38].

\section{B. Student population}

Students taking the Intermediate Mechanics course exhibited a large range of experience and ability in mathematics and physics. Most were physics or engineering physics majors or minors. Students had typically completed introductory physics courses in mechanics, electromagnetism, and optics. Some had a course in modern physics, and many were taking a course in relativity in parallel. Although Intermediate Mechanics was a sophomore level course, the students in this study were fairly equally divided among sophomores and juniors, with some seniors included, leading to a disparity in mathematics backgrounds-about half had already taken a course in differential equations and half took it concurrently. Notably, there was no relationship between a student's class standing (sophomore, junior, or senior) and their mathematics class; some sophomores had already taken differential equations, and some seniors were taking it concurrently. A total of over 50 students were observed as part of the research described in this paper. About 20 are represented in the groups described in this paper, though not all spoke and are therefore not all named in the episodes described below.

In early parts of the Intermediate Mechanics course, students solved first-order, separable differential equations (e.g., $m d v / d t=-b v$ ). For many students, this was an introduction to the method of separation of variables, since their corequisite differential equations course had not yet introduced the topic. However, mastery of the algebra required to separate variables was expected of all students, given the mathematics pre- and corequisites for the course. Separation of variables was simply an extension of algebra that included the treating a differential term $(d v / d t)$ as a fraction.

\section{The problem and its solutions}

As part of our regular classroom instruction, we assigned students several problems on a group quiz given after

$$
\begin{aligned}
& \sum F=m a=m g-b v^{2} \\
& m \frac{d v}{d t}=m g-b v^{2} \\
& \text { Transformcoordinates } \\
& m v \frac{d v}{d x}=m g-b v^{2}
\end{aligned}
$$

FIG. 1. When solving a typical air resistance problem, all student groups sought to separate variables in the last equation shown.

several weeks of instruction on air resistance problems and first order linear differential equations. We asked students to start from Newton's second law to solve the problem of a large object (such as a beach ball) being thrown vertically downward (see the first line of Fig. 1) and to find the velocity of the ball as a function of position. We told them to treat down as positive and that the object was beginning at greater than terminal velocity. They were given no other information, and had to know, for example, that an object of this size would best be described with $v^{2}$ drag forces acting on it. (In one episode below, students did not make this assumption, but this does not affect our data for the purposes of this paper.) All of the observed students successfully moved from the first line to the last line of equations shown in Fig. 1.

The focus of this paper is on what they did at that point. All the groups recognized that the task was to separate variables. To indicate this, we start our transcripts with a student statement to that effect, when possible.

To correctly separate the variables, one might group the terms on the left, $\left(m g-b v^{2}\right)$, and then divide both sides of the equation by the grouped term and multiply both sides of the equation by $d x$. This would move all the terms of $x$ to the right side of the equation and all the terms of $v$ to the other. Or, one might take the metaphorical language of the previous sentence ("move all the terms") and enact it through gestures (as discussed in Ref. [39]). That, or simply write down the equation with separated variables and be done with it.

In the data discussed below, we show that students use several procedural resources. First, there is grouping, in which terms are combined into a single new term. Second, there are the mathematical procedures of subtraction, multiplication, and division. Finally, there is the possibility of moving, which may also require grouping and may involve other actions like pointing and dragging. These gestural elements of moving are not the point of this paper, and are discussed elsewhere [39] in more detail.

We note that all groups eventually accomplished the same task successfully. But, not all immediately solved the problem correctly. In the process of discussing possible solutions, students evaluated the usefulness of the different resources, as will be shown below. 


\section{Choice of student sample}

To observe student reasoning during the group quiz, we video recorded all students as they worked in small groups of 3 or 4 . The students were videotaped with permission, in part to allow the instructor to review their work after the quiz was completed (and assign partial credit where needed) and in part for the purposes of research on how students discussed mathematics in a testing environment. Cameras were placed on tripods at a distance that would allow all the students in the group to be filmed. Because of a lack of cameras, only one camera per table was used.

We observed three kinds of student interactions in our groups. Some groups did not talk while separating variables, so we have no information on what they were doing or their reasoning as they did it. Other groups talked, but did not discuss their reasoning; we give one example, below. The groups we analyzed were those which described their reasoning when isolating the variables in a simple equation.

Video data were collected over the course of three years of instruction. In year 1, we recorded all five groups as they worked on this problem. Of these, two talked about separation of variables in detail. In year 2 , one of the groups provided an excellent example of procedures we had seen in classroom settings but had not yet seen in that detail in the group quiz. In year 3, a further group stood out as providing a unique way of discussing these procedures. We analyzed these four groups as an illustration of the kinds of procedures that students might use when solving this kind of problem. Other groups, not analyzed in this paper, showed similar reasoning or talked less than these particular groups.

\section{E. Constraints on our data selection and analysis}

Small class sizes allowed us to record video data of every group, but restricted us to a small number of groups each year. We gathered data from multiple years in order to have sufficient data to examine patterns, but we do not claim to have observed all possible problem-solving methods or to know what methods may be most common in a broad student population. Rather, as discussed below, our observations begin to build a list of procedural resources that at least some number of students brought to these classes; further research would be required in order to uncover other resources or to study their prevalence among different populations.

Because we gathered data from multiple years of instruction, we increased the variability in students' backgrounds, the specifics of instruction, and other variables. For example, during the time of this study, one author (M. C. W.) taught the course twice and the other (K. E. B.) taught the course once. By combining these data into a single study, we have chosen to leave out course-specific variables in our discussion of students' mathematics use, and instead focus on very general behaviors shared by all the studied groups.
In addition, collecting data during an authentic group activity, as opposed to a group interview, meant that not all groups verbalized their actions as they worked through the targeted task. Some looked off each other's paper without talking at any point, others worked independently and arrived at the same point without discussion, and others discussed only the answer they arrived at, not how they arrived at it. A consequence is that we do not know what all the students in the course did, only those that spoke about their reasoning. The motivations for speaking (such as a strong student needing to explain to a weaker one what is happening at a particular point) might affect the data since such discourse might be more like the explanation of a solution and less the invention of a solution.

Third, the lack of cameras (we had only 5 available to us) meant that we only had one camera to use per group. These cameras were far enough away from the table to include all group members, but were typically too far to allow us to read what students wrote on their papers. Also, they used a screen resolution that did not allow for zooming in more closely while still being able to read what students wrote. This affected our data from year 1; we describe our best interpretations of student work in the episodes below. In years 2 and 3, we asked students to use a whiteboard and markers as they discussed, and we were able to see more of their writing during their discussions.

\section{F. Analysis of data}

Our first data were selected by one author (K. E. B.) while reviewing the year 1 results from all groups. She noticed that students' actions varied from group to group while solving this particular part of the problem. After finding three of the groups to be of interest (while two did not talk), she brought the video of these groups to our 15-person research group meeting. At that point, she presented her preliminary interpretation of the data and a detailed discussion followed. Her first interpretation was not this final version but instead focused on the use of mathematical terminology, did not focus on the gestures students used, and was not yet connected to a resources interpretation. Further interpretations were suggested and discussed, and the analysis was refined as it was put into the context of the larger study. Throughout, we used interaction analysis as described by Jordan and Henderson [40] and a modified version of grounded theory [41,42] in which repeated discussion allowed for the emergence of categories and descriptions of the data that were agreed upon by all participants.

In our choice of data, we came to rely on two different types of observation. We began by focusing on student discourse, attending to students' word choice and their inflection. However, student use of some phrases, like "move this over there" or "this whole term," were ambiguous until we examined the video to describe both the context of the activity and the gestures students used at 
those moments. For a review of gesture analysis [43-47] as it is used in the separation of variables, we refer the reader to a previous publication [39].

In this paper, both discourse and gesture analysis were used to provide evidence for the claim that students' mathematical actions can be thought of as procedural resources.

\section{RESOURCE USE WHEN SEPARATING VARIABLES}

We observed different ways in which students in a group activity combined the procedural resources of grouping, moving, multiplication, and division, and some debate about subtracting, as well. In this section, we present several episodes (transcripts and description of events) to illustrate variations in student actions; in the following discussion, we analyze the consequences of variations in student activities and thinking.

We suggest that for a typical physicist, separating variables in this problem might be a necessary but trivial step in working through the problem. This appeared to be the case for one of the stronger students in the class, alias Phil. While solving the problem, Phil did not verbalize any explanation of his steps; he simply arrived at an answer, as follows:

1 Phil: OK, now we want to separate variables. We have $m v d v$ over $m g$ minus $b v$ squared equals [makes noises while writing] uhh, $d x$.

(We number lines of transcript sequentially in this paper to help in the discussion of group interactions.)

Though we cannot see what is on the page, we hear him speak the correct answer, arrived at with no explicit algebraic steps indicated. We believe that students eventually arrive at this level of expertise, using the procedure as a single resource, much like addition and multiplication become basic resources (e.g., primitive [21]) to a student. We return to this point in the discussion.

\section{A. Sarah divides and multiplies to separate}

For three students, Sarah, Paul, and Tim, separating variables was not trivial. These students worked individually on the problem while occasionally comparing their solutions. Sarah first silently completed the separation of variables on her own paper and then helped her classmates Paul and Tim to catch up. In this episode, we attend primarily to Sarah as she interacts first with Phil and later with Tim. We italicize words in the transcript which we refer to later in the discussion or analyze in terms of resources.

2 Sarah: Alright, so where are you guys at?

3 Paul: I'm still trying to separate it.

4 Sarah: OK, um, one of the easiest ways is dividing the entire thing by this side, and then multiplying both sides by $d x$.
5 Paul: Yeah, yeah.

6 long pause-more than a minute

7 Sarah: Ok, alright. What are you doing right now?

8 Tim: Alright, so we're at this point, right? Weve got $m v$ $d v d x$ is equal to $m g$ minus $b v$ squared.

9 Sarah: Mm-hm.

10 Tim: Alright. So what you're doing is just bringing this $d x$ over?

11 Sarah: You bring the dx over and then divide both sides by this entire, um, expression. So it becomes, um, $m v$ $d v$ over $m g$ minus $b v$ squared.

We analyze line 4 first. Sarah responded to Paul's line 3 statement about separating variables in the equation by naming two procedures-dividing the whole equation ("the entire thing") by one side of the equation ("this side") to get all the velocity terms on one side and then multiplying by $d x$ to get the position terms on the other side of the equation. When she said "the entire thing", she underlined both the left and right sides of the equation in a single gesture, and when she said "this side", she circled the $m g-b v^{2}$ on the right side of the equation. We interpret this to mean that she was carrying out algebraic steps (dividing) on both sides of the equals sign, being careful to define by grouping what she was dividing with. For Sarah responding to Paul, separation happened by grouping one side of the equation, followed by dividing and then multiplying.

Later in the episode, Sarah helped Tim with his work as Paul observed their work silently (Paul had not "separated it" after his comment in line 5). In line 8, Tim stated the equation that all seemed to be working with. In line 10, he suggested something different from Sarah, namely, "bringing this $d x$ over" to the other side of the equation. In line 11, Sarah affirmed that this idea of bringing over was equivalent to the multiplication she described to Paul earlier (or at least consistent with her act of multiplying), and then repeated her statement about division. As before, Sarah's ambiguous words about "this entire... expression" were accompanied by a vigorous circling gesture (during the word "entire") which we believe represents the procedure of grouping the several terms she was referring to (Tim's " $m g$ minus $b v$ squared" and not "the entire thing" that she described when talking to Paul). She concluded line 11 with a statement of the separated equation, $m v d v /\left(m g-b v^{2}\right)=d x$.

In both cases, the grouping procedure preceded the division procedure; mathematically speaking, it was a necessary step before division could be used to successfully separate variables. But, in both cases, the verbal and gestural evidence of grouping came after the use of "division" in speech.

As with Phil (line 1), these students wished to separate the terms in the equation, but they described many more procedures to do so. We see Sarah grouping and dividing and then multiplying to arrive at a separated equation. We 


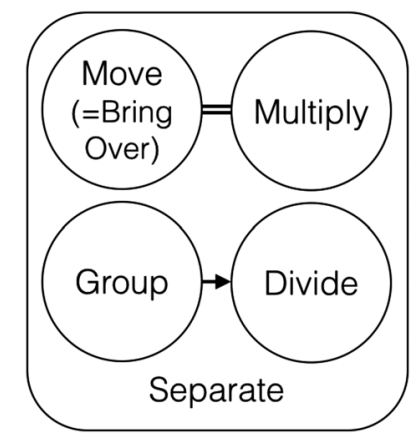

FIG. 2. Sarah's procedure of separation contains multiple procedures. These include formal mathematical operations and explicit activity to group terms in the equation appropriately. She also indicates an equivalence between a description of moving and the mathematics of multiplication.

also see Tim moving terms by "bringing ... over" a term from one side to the other. (We discuss other examples of moving in more detail below.)

Through her interactions with her group, Sarah shows that one way of separating is to think in terms of mulitplying and dividing while being careful about grouping terms that are to be part of the division. We represent the collection of Sarah's resources in a graph where the process of separation (which for Phil was a single action) in this example is made up of multiple procedures (see Fig. 2). In the graph, we indicate that grouping precedes dividing by using an arrow that the one leads to the other. We also indicate that Tim's moving by "bringing... over" is equivalent to Sarah's multiplying by using a double line like an equals sign.

\section{B. Students move to separate}

In a separate episode, two students, Simon, who was in control of the whiteboard marker, and Dan, who dominated the conversation, discussed separation of variables quite differently from Phil or Sarah. (Two other students in the group did not speak during this episode.) We have described the use of gesture in this episode elsewhere [39]. Here, we discuss the procedural resources used by the students. As before, we mark words we will return to by italicizing them. We use [square brackets] to describe gestures essential to understanding the text.

12 Simon: So then we're gonna shuffle things around

13 Dan: Yes. $d x$ over $m$ -

14 Simon: $d$ what? $d x$ over -

15 Dan: $d x$ over $m$

16 Simon: You mean... $d \ldots d$ ?

17 Dan: I just did this whole thing now, this exact same problem.

18 Simon: Are you moving $d x$ over there?

19 Dan: Yeah, you move $d x$ over there, and them [his fingers bracket the $m g-c_{2} v^{2}$ on the white board] over there and this $[\mathrm{m}]$ over there
20 Simon: Let's do it like this first.

21 Dan: It doesn't matter...

22 Simon: $m v d v$ equals $m g$ minus $c$ two $v$-squared $d x$

23 Dan: Yeah, if you want to write down that step.

24 Simon: And then we get the $v$ on the other side

25 Dan: We move this [fingers bracket $m g-c_{2} v^{2}$ ] over there and that [points at $d x$ ] over here.

26 Simon: Move the one with $v$ over there?

27 Dan: Yeah. Move this whole term [fingers bracket again] over there.

28 Simon: $m v$ over $m g$ minus $c$ two $v$ squared equals $d x$.

Even as Simon very formally wrote out all the steps required to separate variables, Dan simply "move[d]" terms around on the white board. But, Dan and Simon showed a different set of procedures than Sarah did in talking to Paul and Tim. They moved terms, in response to Simon's desire to shuffle things around, but never spoke of dividing or multiplying.

There were several moments of ambiguity which suggest that Simon was unsure of what to do until Dan clarified. For example, in line 26, Simon asked which terms to move, and asked if he should only move the one term with $v$. Dan responded by making clear he meant the whole term, and Simon then accurately wrote down " $m g$ minus $c$ two $v$ squared" (line 28). The gestures used by Dan at this point were consistent with other gestures he made in line 19: he bracketed the desired terms with fingers on either side. This bracketing gesture helped make sense of "them" (line 19) and "this whole term over there" (line 27) and stood in contrast to him simply pointing at "this" (line 19) when referring to the $m$ in the equation.

Like Sarah, Dan was using gestures to indicate the grouping of terms.

Where Sarah had grouped, divided, and showed an equivalence between multiplication and moving (Fig. 2), Dan was focusing only on grouping and moving without naming mathematical operations. Where Tim had only made an equivalence between multiplication and moving, Dan used moving to carry out the procedures of both multiplication (the $d x$ in line 19) and division (the $m g-c_{2} v^{2}$ and $m$ later in line 19).

As before, we make a graph of the resources used by one student in the group. We describe Dan in Fig. 3, because he does most of the work here. In the resource graph, we see that separating only consists of grouping which precedes moving, like with Sarah.

\section{Subtraction isn't a good choice}

Our third episode of students separating variables contains a debate about which procedures are to be used. The students know that they must get all the terms of one sort to one side of the equation and all the others to the other side, but they are not sure how to do so. The students arrive at the correct answer, but their work is interesting to us because 


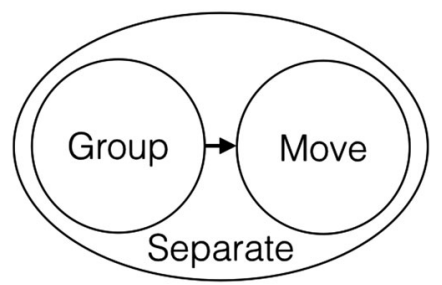

FIG. 3. Dan's resource of separation contains the procedures of grouping and moving.

they explicitly evaluate the use of an incorrect procedure and decide not to use it.

29 Jared: Now we can separate, right?

30 Keith: Yes.

31 Jared: Do, we, do we want to pull the whole entire term over, or-like divide by an entire-

32 Keith: I think we want to divide-

33 Brian: What term are we gonna have on one side? Just the $v$ ?

34 Jared: I think we want to pull this entire term over, divide by it.

35 Brian: Yeah.

36 Jared: Cause if, cause if-

37 Ann: Well, when-

38 Jared: Because if you subtract you're gonna have a zero, so there's no way to separate the variables after that.

39 Jared: (after a silence) So it's going to be $m$ over $m g$ minus $b v$ squared $d v d x$ equals one. Which is why we wanted to, because we wanted to have the one on the other side instead of a zero. So $m d v$ over $m g$ minus $b v$ squared equals $d x$. Right?

Like Sarah and Dan, Jared used gestures to group terms and talked about moving terms, though he was more like Sarah in that he also talked about division, which Dan had not talked about.

In line 31, Jared introduced the idea of division, a step that was supported by Keith in line 32. Jared was clear that he was talking about "the whole entire term" (line 31), indicating the " $m g$ minus $b v$ squared" (from line 39) by drawing exaggerated parentheses in the air above his sheet of paper. This stands in contrast to Brian's question, in line 33, about which terms to divide by, the "term [with] just the $v$ ?" Brian's question was akin to Simon's question to Dan in lines 26 and 27. Jared responded by repeating his desire (like Sarah and Dan in their interactions) to use "this entire term", (line 34).

We note that Jared, in line 34, drew an equivalency between "pull[ing] this entire term over" and "divid[ing] by it." For Jared in this situation, moving was like division, but not like multiplication, as it was with Dan.

Later, Jared contrasted the procedure of pulling or division with the procedure of subtraction (line 38), which would lead to a zero and an inability to continue separating variables. We note that subtraction leading to a zero requires everything on that side of the equation to

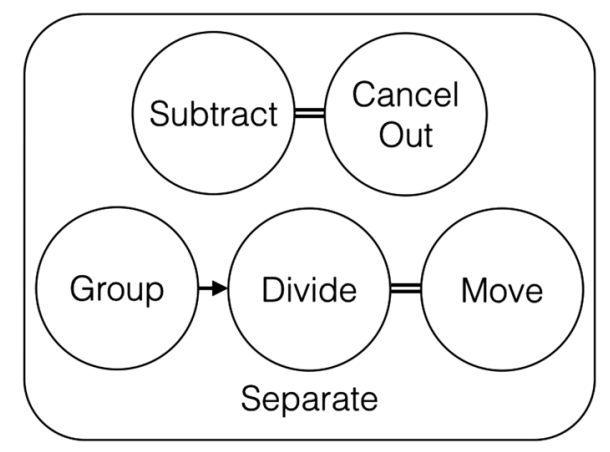

FIG. 4. Jared's procedure of separation included grouping and division and an explicit statement that subtraction was an inappropriate procedure.

be subtracted, not just the $v$ term, telling us that Jared was working with the grouped set of terms on the right side of the equation. Jared elaborated on his thinking about subtraction in line 39 , talking about needing a "one on the other side instead of a zero." Having a 1 allowed for the implicit procedure (multiplication or moving is not specified) of the $d x$ moving from the denominator on the left to the numerator on the right. A zero would not have allowed that (e.g., multiplying anything by zero leads to zero, so subtraction must be the wrong step to take).

We represent Jared's use of separation in the graph shown in Fig. 4. As before, grouping is needed before one can divide, which in this case is equivalent to moving by "pulling over" from one side of the equation to the other. In contrast to Sarah, though, Jared suggests grouping before he mentions dividing, in line 31. Subtraction is considered, but it would lead to a "zero" which we describe as a canceling out because Jared describes it as ending the possibility of further work (in line 38). The idea is not pursued further by Jared or the others. Finally, though the $d x$ term ended up on the other side of the equation from where it began, the group does not discuss multiplication or moving for it to have done so. As a result, multiplication is not part of the graph.

\section{Subtraction and division without grouping}

In the final episode of this paper, students used the same resources as have been named before, but some considered subtraction and division without also considering grouping. In this example, students are working with a slightly different equation, $m d v / d t=m g-c v$, valid for $v$-dependent drag forces. Because of the length of the full discussion of separation of variables, we highlight only a one minute-long segment of the discussion before the students resolved their problems.

In our episode, Charlie was scribe, setting up the situation, while Bill and Derrick were the ones debating what to do. As the episode started, Charlie had written an 
incomplete equation on a whiteboard, but his head blocked the written work from our camera.

40 Charlie: That's why I tried to get the $c v$ over there

41 Bill: It could be one over, it could be one... one over, $c v$ and one over... no...

42 Bill: (a short moment later) Um, leave $c v$, leave it right where it is, move this [pointing to $m g$ ] over, take this [pointing to the $d t$ in $d v / d t$ ] and put it over there and that would be one over $c v$.

43 Derrick: Could you divide it by this whole side [brackets $m g-c v$ with his fingers] and multiply it by $d t$ ?

44 Bill: Just subtract the $m g$

45 Derrick: Really? [His fingers bracket $m g-c v$ again]

46 Charlie: Wait, I'm trying to do, like, two different things at once, but let me just erase, let me do it, let me do it!

Charlie, who started talking about the $c v$ alone, recognized by the end that the two ideas (Bill's and Derrick's) needed to be resolved; we do not quote from the lengthy discussion that followed but note that they did, eventually, follow Derrick's suggestion.

We note similarities in this interaction and the previous ones. As a reminder: Phil used separation without explanation, Sarah grouped terms she manipulated mathematically while assisting Paul and Tim, Simon and Dan grouped and moved terms without using any mathematical language, and Jared considered subtraction while grouping, moving, and dividing to separate variables. Bill used moving to describe both multiplication (line 42) and subtraction (as we draw the parallel between line 42 and 44). Like Brian (line 33) and Simon (lines 24 and 26), Charlie and Bill were not grouping terms and instead were working with either the $c v$ or the $m g$ alone. In contrast, in lines 43 and 45, Derrick suggested dividing by "this whole side" with gestures that clearly indicated that he meant both terms, like Sarah (lines 4 and 11), Dan (lines 19, 25, and 27), and Jared (lines 31 and 34). Like Sarah (lines 4 and 11), Derrick grouped with gestures, indicated the grouping by talking about "this whole side", and multiplied, rather than moved, to have the differential term from the denominator on one side be in the numerator on the other side of the equation.

From this example, we observe the distinct procedures of grouping, subtraction, division, and multiplication. We observe Bill struggling to divide by $c v$ (lines 41 and 42 , leading to the one over $c v$ ) or subtract by $m g$ (line 44 , and possibly also 42) while not grouping. Also, we observe Derrick grouping and dividing and multiplying, without using the idea of subtraction.

We summarize Bill and Derrick's very different approaches in two graphs in Fig. 5. In Bill's graph, we indicate the implied equivalence between moving and multiplication (of $d t$ ) as well as the more explicit equivalence of moving and subtracting.

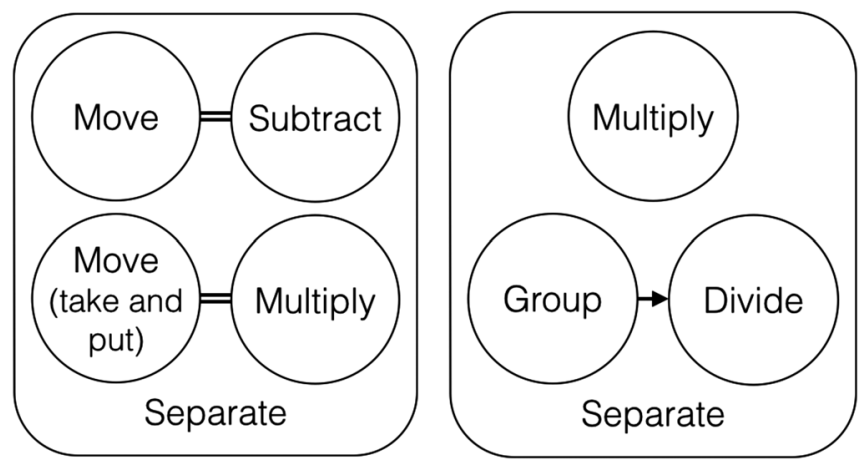

FIG. 5. Bill's two uses of moving without grouping are in contrast to Derrick's use of multiplication and division after grouping.

\section{DISCUSSION}

In these four episodes, as well as the single statement by Phil in line 1, we observe a variety of mathematical actions including subtraction, multiplication, and division, as well as moving and grouping. All have been used with the purpose of separation of variables, a necessary step in the particular problem that students were solving.

In this section, we discuss why these different mathematical actions can be thought of as procedural resources, highlight the importance of the grouping resource, and discuss the issue of expertise in separation. Finally, we discuss implications for teaching.

\section{A. Theoretical issues around procedural resources}

Earlier, we provided two different sets of criteria that help define resources. We find that the mathematical actions described in this paper are consistent with these criteria.

\section{Mathematical actions as procedural resources}

Consistent with Sayre and Wittmann [28], we find ample evidence that the mathematical actions are "individual" and "reusable" parts of problem solving. They were not only used by multiple students, but by individual students at multiple times during a single episode. We find support for the idea of activation, namely, that the ideas were active in a given setting. We have ample evidence from our classes to know that these students could all group, divide, and so on. We find that some of these ideas were activated and some were not, in this setting. Furthermore, we find that the actions were connected to other actions, such as when grouping was named as a necessary element before one could divide. Finally, we observe that there was some level of internal structure to a given action. Where Phil may have had a single action of separation, the other students indicated that several resources were needed; we can think of separation as being made up of these resources, and return to this point below. In a second example, the resource of moving seemed to have elements of pointing and 
dragging associated with it. By Sayre and Wittmann's criteria, then, these mathematical actions are resources.

Consistent with Scherr's analysis of the knowledge-inpieces model [17], we also find ample evidence that these mathematical actions were mutually independent, contextdependent, and indeterminate. They were mutually independent as can be seen from the ways that the students used the individual actions in a variety of orders and invoked them separately from each other. Each group did something slightly different with these few mathematical actions. They were context-dependent in that some actions were useful in this setting, but others (for example, subtraction) were not going to lead to a solution of the problem. Finally, there was nothing inherently incorrect about these mathematical actions. Subtraction is subtraction, regardless of context. The idea itself was not correct or incorrect, only its use.

By naming mathematical actions "procedural resources," we expand the discussion of the use of mathematics in physics and allow some of the machinery associated with the resources framework to be associated with this area of study. We can talk about transfer, for example, while connecting to previous analysis of these issues [2]. We can analyze activation and how it relates to epistemological concerns [14-16]. In sum, identifying mathematical moves as procedural resources allows us to carry out analysis based on previous work on resources.

\section{Modeling resources as parts of resources}

One particular way we can make use of the resources framework is to help make sense of the scale and grain size of these procedural resources and how resources come together to form new resources. Here, we address Hammer et al. as they explain, "locally coherent sets of resources may, over time, become established as resources in their own right" [2], seeking to bring detail to their statement.

The data suggest that the students' locally coherent set of resources were different. For Phil, there was a single resource (as there probably was for Sarah and Dan, as well, were they not working in a group setting). Sarah and Derrick used gestures to group and mathematical procedures to reorganize their equation. Dan used only gestures to both group and move. Jared used gestures and mathematics, but took the additional step of explicitly excluding the procedure of subtraction from the act of separation (at least in this context, because in others it might be perfectly reasonable). It is possible that Phil's single action of separation is simply the reification [31,32] of locally coherent sets of resources.

The variety of local coherences suggests that there is no single path toward learning separation of variables, and that many different paths can lead to separation as a resource in its own right. We do not have the data to determine how the use of these resources develops in time. Would Jared eventually act like Sarah, who might later act like Dan, until they are all acting like Phil? Our data do not provide an answer. Further work would be needed to determine the details of locally coherent resources becoming resources of their own.

Our work suggests that coherence may be determined by one's group interactions, since Sarah and Dan both seemed to engage in discussion only because of their group members. Further study would be required in this area of resource creation.

Finally, our work suggests that resource compilation, reification, or creation is a process that requires further study. We cannot expect experts to carefully carry out every step of every problem; at some point, facility with new ideas is needed. At the same time, we expect experts to be able to "unpack" resources. We suggest that Sarah and Dan are engaged in such an unpacking, as they interact with their groups.

\section{B. The importance of particular resources: Grouping}

Grouping played an essential role in all four episodes Students in each episode (Tim, Simon, Brian, Bill, Charlie) questioned which terms one must move or divide by. The task of moving all of one variable to one side of the equation would suggest that one only move the terms that include that variable. Moving both the $m g$ and the $b v^{2}$ together is not the obvious choice, unless one thinks ahead in the problem. The particular reason that grouping is needed is because the typical next step (of multiplying by the $d x$ ) would lead to mixed terms if one only subtracted or divided by $b v^{2}$.

Some students talked about dividing or moving before they defined what they were dividing by or moving. Sarah (lines 4 and 11) and Derrick (line 43) talked about dividing and only then about grouping. Jared (line 31 and 24) wanted to "pull the whole term over," so the pull preceded the definition of what he was pulling. Maybe these students knew what they were moving or pulling or dividing by before they spoke. Our data cannot give us further information.

A different solution lies in a different order of operations. Rather than moving the $m g-b v^{2}$ term first, Dan created a mathematical solution that created a grouped term to manipulate next. Dan (line 19) moved the $d x$ first, which basically required grouping as it came to multiply $\left(m g-b v^{2}\right)$, creating parentheses which indicated the grouping that happened (as Simon wrote in line 22). The difference in order made the problem of identifying grouped terms easier.

We note that grouping typically happened with a gesture. Dan and Derrick used their thumb and a finger to bracket $m g-b v^{2}$, indicating what they were about to move or divide by. Sarah circled the two terms. Jared indicated parentheses around the terms, much like Simon wrote. For all these students, gesture were an important act in grouping. From our data, we cannot tell if this was because of the role of gesture in explanations in a group environment or if 
it is related to something more fundamental about the role of gesture in our thinking.

\section{Resources and expertise}

We have already mentioned the issue of unpacking an idea as a type of expertise and suggested that Sarah and Dan showed this level of expertise. In this section, we discuss further issues that relate to expertise.

Given that all the groups successfully separated variables, we note large differences in their ease of carrying out the procedure. Phil flew through the task. Dan was only slowed down by Simon's writing. Sarah could have moved on easily, were it not for helping Paul and Tim. Jared's group struggled. Derrick's group was mired in the longest discussion of all these groups, mostly because they were not grouping terms correctly.

Our discussion of these observations is tied to the number of ideas they had to keep in mind, and the limited ability of working memory to consider too many ideas at once. Derrick's group was caught by not having included a necessary resource. Jared's group had many questions and procedures to consider: what is the consequence of subtraction? a canceling that is not desired; is pulling over the same as division? and what do we divide by? To think of all those things and consider them carefully would slow any group down. Sarah, in contrast, had a clear task to convey and did so twice. Phil just solved the problem and moved on, treating the act of separation as a single procedure.

We expect of our students that they learn to solve problems quickly, including carrying out certain mathematical steps automatically. This might be a kind of quick pattern matching, or it might be the reification of locally coherent sets of resources into a single resource. If we think of resources as chunks of working memory, as suggested above, we suggest that a single chunk of separation can be worked with easily (as Phil does), but that the use of additional procedural resources requires additional work and slows students down (as Dan and Sarah seem to be slowed by their groups or Jared by his many considerations). Of course, part of the slowness comes from having to name and elaborate what is meant by the procedures.

A different kind of expertise can be found in Jared, in a way that is not observed in the other students. Jared considered more than one method for solving the problem and was able to account for why this method was incorrect. That kind of thinking required that he be working with the sub-pieces of separation rather than the resource itself. This second definition of expertise is about the ability to analyze situations in detail and understand not just what works but also what doesn't.

The resources analysis provided in this paper allows for multiple forms of expertise to be discussed within the theoretical framework.

\section{Implications for teaching}

In this paper, we have looked closely at students solving one part of a many part problem. The differences in student actions suggest some of the difficulty of teaching students about a topic like separation of variables. Given the importance of the topic in intermediate and advanced physics (including when solving partial differential equations), and given the role of algebraic manipulation in all of physics, the insights gathered from this paper have implications for our teaching.

First, applying a resources perspective to mathematical actions expands the implications of pedagogical suggestions made in the literature [23,48]. On a theoretical level, we have not engaged with the implication that mathematical actions are thoughts, an issue which would require a longer exploration of work in the learning sciences (see, for example, the work of Nemirovsky and collaborators [49-52]). Taking this perspective would provide us with a foundation for modeling group interactions and group reasoning, a useful tool when our data come from authentic classroom group activities, like in this paper. On a more concrete level, we should expect to find variety in students' mathematical work much like we find it in their conceptual reasoning, and we should also be providing them with problems to solve in which a greater variety of mathematical reasoning is possible.

Second, students have a multitude of ways of correctly solving this problem. Taken more generally, physics problems typically have multiple solutions. As an instructor, it is important to recognize all these solution pathways and to recognize their value and their shortcomings. It may be that one is more effective than the other, such as Dan effectively multiplying by $d x$ before dividing by $m g-b v^{2}$. Knowing what to suggest to a student at a given moment requires an understanding of the variety of student thinking as has been provided in this paper.

Third, our students had the most difficulty with grouping terms. Where other procedural resources in this paper had formal mathematical names (subtract, divide, etc.), grouping has associated mathematical representations (using parentheses correctly) but does not have as formal a name. Simply being aware that students struggle with this idea might be of help to an instructor, or a more direct intervention might be developed.

\section{CONCLUSION}

The mathematical procedure of separation of variables is common to upper division physics and is a common tool used when solving first order differential equations. Students begin to learn the procedure in their post-introductory courses. In our study, the first course in which students used the idea in physics was Intermediate Mechanics.

In this paper, we have argued that there are multiple procedural resources that can be brought into problem 
solving, and that these resources are used in different combinations by different groups of students. We consider those resources which are procedural, rather than conceptual, because we can more easily observe the use of a specific procedure than we could infer the concepts a student might have when carrying it out. These procedural resources fulfill the criteria set forth by Sayre and Wittmann [28] and Scherr [17].

We have discussed the need for creating new resources (or compiling or reifying existing networks of resources) as a way of making problem solving more efficient, an expertlike behavior. We have suggested that the episodes we present are pathways to the creation of this new resource, separation, and have shown one example (Phil, in line 1) of what the use of this resource might look like. We have also shown what it looks like when students are not using a single resource to separate variables, including when they are evaluating which resources to use and choosing not to use some. In the process, we have discussed how multiple forms of expertise can be described within a resources framework.

Extensions of this work might be found in several areas. We have suggested two. First, if we consider mathematical actions as a kind of thought, we raise questions about the nature of knowledge in a social and material context, a topic we have discussed previously $[39,53,54]$. Second, an awareness of the variability in students' mathematical thinking is an essential element of advanced physics instruction. In particular, focusing on the utility of a resource, rather than its correctness, allows for a refinement of our model of teaching [48]. Speer and colleagues have investigated teacher understanding of the ideas that students bring to the college-level classroom for example $[55,56]$. For instance, a student solving a similar problem on an examination (as described in Ref. [57]) used the variational principle to solve this problem-a perfectly valid and correct approach, though one rarely used by physics faculty, in our experience. Other extensions might be to investigate the use of procedural resources over time and in new contexts, to study the interactions of procedural and epistemological resources, or to define how procedural resources are parts of larger networks of ideas, such as in coordination class theory $[5,58,59]$.

Using the resources framework, we are able to make sense of students' differences while also showing what they have in common. Our contribution, then, is both to the knowledge of students' uses of mathematics in a physics classroom and to the development of a model of knowledge that plays a central role in our research community.

\section{ACKNOWLEDGMENTS}

A previous version of this analysis was published as Ref. [9]. In returning to that analysis, we have refined several points. Our thanks to Brandon Bucy and Kate Hayes for assistance in videorecording and Lauren BarthCohen, Dan Capps, and Laura Millay for their help in preparing this manuscript. This work was supported in part by the National Science Foundation under Grants No. DUE-0441426, No. DUE-0442388, and No. REC0633951, and No. MSP-0962805.
[1] D. Hammer, Student resources for learning introductory physics, Am. J. Phys. 67, S45 (2000).

[2] D. Hammer, E. F. Redish, A. Elby, and R. E. Scherr, Resources, Framing, and Transfer, in Transfer of Learning: Research and Perspectives, edited by J. P. Mestre (Information Age Publishing, Charlotte, NC, 2005), pp. 1-26.

[3] E. F. Redish, A Theoretical Framework for Physics Education Research: Modeling student thinking, in Proceedings of the International School of Physics "Enrico Fermi," Course CLVI: Research on Physics Education, edited by E. F. Redish and M. Vicentini (IOS Press, Amsterdam, 2004), pp. 1-56.

[4] M. S. Sabella and E. F. Redish, Knowledge organization and activation in physics problem solving, Am. J. Phys. 75, 1017 (2007).

[5] M. C. Wittmann, The object coordination class applied to wavepulses: Analysing student reasoning in wave physics, Int. J. Sci. Educ. 24, 97 (2002).

[6] M. C. Wittmann, Using resource graphs to represent conceptual change, Phys. Rev. ST Phys. Educ. Res. 2, 020105 (2006).
[7] K. Hayes and M. C. Wittmann, The role of sign in students' modeling of scalar equations, Phys. Teach. 48, 246 (2010).

[8] M. C. Wittmann and K. E. Black, When basic changes to a solution suggest meaningful differences in mathematics, edited by N.S. Rebello, P. V. Engelhardt, and C. Singh, AIP Conf. Proceedings, Vol. 1413 (AIP, Melville, NY, 2012), pp. 93-96.

[9] K. E. Black and M. C. Wittmann, Procedural Resource Creation in Intermediate Mechanics, edited by M. Sabella, C. Henderson, and C. Singh, AIP Conf. Proc. No. 1179 (AIP, Melville, NY, 2009), pp. 97-101, ISBN 9780735407206.

[10] M. C. Wittmann and K. Black, Choosing integration methods when solving differential equations, in Proceedings of the International Conference on the Learning Sciences, Vol. 3 (International Society of the Learning Sciences, Utrecht, Netherland, 2009), pp. 155-156.

[11] K. E. Black and M. C. Wittmann, Epistemic Games in Integration: Modeling Resource Choice, edited by L. Hsu, C. Henderson, and L. McCullough, AIP Conf. Proc. No. 951 (AIP, Melville, NY, 2007), pp. 53-56. 
[12] C. Kieran, Learning and teaching algebra at the middle school through college levels, in Second Handbook of Research on Mathematics Teaching and Learning, edited by F. K. Lester (Information Age Publishing, Charlotte, NC, 2007), Vol. 2, Chap. 16, pp. 707-762.

[13] C. Kieran, The Learning and Teaching of School Algebra, in Handbook of Research on Mathematics Teaching and Learning, edited by D. A. Grouws (Macmillan, New York, 1992), Vol. 2, pp. 390-419.

[14] A. Elby and D. Hammer, On the Substance of a Sophisticated Epistemology Sci. Educ. 85, 554 (2001).

[15] D. Hammer and A. Elby, Epistemological Resources, in Fourth International Conference of the Learning Sciences, edited by B. Fishman and S. O'Conner-Divelbiss (Lawrence Erlbaum Associates, Mahwah, NJ, 2000), pp. 4-5.

[16] D. Hammer and A. Elby, On the Form of a Personal Epistemology, in Personal Epistemology: The Psychology of Beliefs about Knowledge and Knowing, edited by B. K. Hofer and P. R. Pintrich (Lawrence Erlbaum Associates, Mahwah, NJ, 2002).

[17] R. E. Scherr, Modeling student reasoning: An example from special relativity, Am. J. Phys. 75, 272 (2007).

[18] M. Minsky, The Society of Mind (Simon and Shuster, New York, 1985).

[19] D. Klahr and R.S. Siegler, The Representation of Children's Knowledge, in Developmental Psychology: An Advanced Textbook, 3rd ed. edited by M. H. Bornstein and M. E. Lamb (Lawrence Erlbaum Associates, Hillsdale, NJ, 1992).

[20] J. Minstrell, Facets of students' knowledge and relevant instruction, in Research in Physics Learning: Theoretical Issues and Empirical Studies, Proceedings of an International Workshop, Bremen, Germany 1991, edited by R. Duit, F. Goldberg, and H. Niedderer (IPN, Kiel, 1992), pp. 110-128.

[21] A. A. DiSessa, Towards an epistemology of physics, Cognit. Instr. 10, 105 (1993).

[22] B. L. Sherin, Ph.D. dissertation, University of California, Berkeley, 1996, unpublished.

[23] D. Hammer, Misconceptions or P-Prims: How may alternative perspectives of cognitive structure influence instructional perceptions and intentions?, J. Learn. Sci. 5, 97 (1996).

[24] D. Tirosh, R. Staby, and S. Cohen, Cognitive conflict and intuitive rules, Int. J. Sci. Educ. 20, 1257 (1998).

[25] B. L. Sherin, How students understand physics equations, Cognit. Instr. 19, 479 (2001).

[26] G. A. Miller, The magical number seven, plus or minus two: Some limits on our capacity for processing information, Psychol. Rev. 63, 81 (1956).

[27] C. von Aufschnaiter, S. von Aufschnaiter, C. v. Aufschnaiter, and S. v. Aufschnaiter, Theoretical framework and empirical evidence of students' cognitive processes in three dimensions of content, complexity, and time, J. Res. Sci. Teach. 40, 616 (2003).

[28] E. Sayre and M. Wittmann, Plasticity of intermediate mechanics students coordinate system choice, Phys. Rev. ST Phys. Educ. Res. 4, 020105 (2008).
[29] E. F. Redish, R. E. Scherr, and J. Tuminaro, Reverse engineering the solution of a simple physics problem: Why learning physics is harder than it looks, Phys. Teach. 44, 293 (2006).

[30] A. Sfard, Operational origins of mathematics objects and the quandary of reification - the case of function, in The Concept of Function: Aspects of Epistemology and Pedagogy, 25th ed. edited by G. Harel and E. Dubinsky (Mathematical Association of America, Washington, DC, 1992), pp. 59-84.

[31] A. Sfard, On the dual nature of mathematical conceptions: Reflections on process and objects as different sides of the same coin, Educ. Stud. Math. 22, 1 (1991).

[32] A. Sfard, Reification as the birth of metaphor, Tijdschrift Voor Didactiek Der Beta-Wetenschappen 13, 5 (1995).

[33] B.S. Ambrose, Investigating student understanding in intermediate mechanics: Identifying the need for a tutorial approach to instruction, Am. J. Phys. 72, 453 (2004).

[34] B.S. Ambrose and M. C. Wittmann, Intermediate Mechanics Tutorials (2007), URL http://faculty.gvsu .edu/ambroseb/research/IMT.html.

[35] B.S. Ambrose, Incorporating a tutorial approach in an advanced mechanics course for physics majors, The Announcer 33, 124 (2003).

[36] L. C. McDermott, P. S. Shaffer, and The Physics Education Group at the University of Washington, Tutorials in Introductory Physics (Prentice Hall, Upper Saddle River, NJ, 2002).

[37] R. N. Steinberg, M. C. Wittmann, and E. F. Redish, Mathematical Tutorials in Introductory Physics, in AIP Conference Proceedings 399 (Springer, New York, LLC, Secaucus, NJ, 1997), Vol. 399, pp. 1075--1092, ISBN 1563966980 , .

[38] M. C. Wittmann, R. N. Steinberg, and E. F. Redish, Activity-Based Tutorials Volume 1: Introductory Physics (John Wiley \& Sons, Inc., New York, 2004).

[39] M. C. Wittmann, V. J. Flood, and K. E. Black, Algebraic manipulation as motion within a landscape, Educ. Stud. Math. 82, 169 (2013).

[40] B. Jordan and A. Henderson, Interaction analysis: Foundations and practice, J. Learn. Sci. 4, 39 (1995).

[41] B. Glaser and A. Strauss, The Discovery of Grounded Theory: Strategies for Qualitative Research (Aldine, Chicago, 1967).

[42] A. Strauss and J. Corbin, Basics of Qualitative Research: Grounded Theory Procedures and Techniques (Sage, Thousand Oaks, CA, 1990).

[43] W.-m. Roth and M. Welzel, From activity to gestures and scientific language, J. Res. Sci. Teach. 38, 103 (2001).

[44] W.-M. Roth and D. V. Lawless, Scientific investigations, metaphorical gestures, and the emergence of abstract scientific concepts, Learn. Instr. 12, 285 (2002).

[45] W.-M. Roth, From action to discourse: The bridging function of gestures, Cognit. Syst. Res. 3, 535 (2002).

[46] D. Givry and W.-M. Roth, Toward a new conception of conceptions: Interplay of talk, gestures, and structures in the setting, J. Res. Sci. Teach. 43, 1086 (2006).

[47] R. E. Scherr, Gesture analysis for physics education researchers, Phys. Rev. ST Phys. Educ. Res. 4, 010101 (2008). 
[48] D. Hammer, Discovery learning and discovery teaching, Cognit. Instr. 15, 485 (1997).

[49] R. Nemirovsky, C. Rasmussen, G. Sweeney, and M. Wawro, When the classroom floor becomes the complex plane: Addition and multiplication as ways of bodily navigation, J. Learn. Sci. 21, 287 (2012).

[50] R. Hall and R. Nemirovsky, Introduction to the special issue: Modalities of body engagement in mathematical activity and learning, J. Learn. Sci. 21, 207 (2012).

[51] R. Nemirovsky, Three conjectures concerning the relationship between body activity and understanding mathematics, in Proceedings of the 27 PME Conference, University of Hawaii (University of Hawaii, Honolulu, 2003), pp. 105-109.

[52] R. Nemirovsky and F. Ferrera, Mathematical imagination and embodied cognition, Educ. Stud. Math. 70, 159 (2009).

[53] V. J. Flood, F. G. Amar, R. Nemirovsky, B. W. Harrer, M. R. M. Bruce, and M.C. Wittmann, Paying attention to gesture when students talk chemistry: Interactional resources for responsive teaching, J. Chem. Educ. 92, 11 (2015).
[54] R. E. Scherr, H. G. Close, E. W. Close, V. J. Flood, S. B. McKagan, A. D. Robertson, L. Seeley, M. C. Wittmann, and S. Vokos, Negotiating energy dynamics through embodied action in a materially structured environment, Phys. Rev. ST Phys. Educ. Res. 9, 020105 (2013).

[55] N. M. Speer and J. F. Wagner, Knowledge needed by a teacher to provide analytic scaffolding during undergraduate mathematics classroom discussions, J. Res. Math. Educ. 40, 530 (2009).

[56] N. M. Speer, K. D. King, and H. Howell, Definitions of mathematical knowledge for teaching: Using these constructs in research on secondary and college mathematics teachers, J. Math. Teacher Educ. 18, 105 (2014).

[57] M. C. Wittmann and K. E. Black, Visualizing changes in pretest and post-test student responses with consistency plots, Phys. Rev. ST Phys. Educ. Res. 10, 010114 (2014).

[58] A. A. DiSessa and B. L. Sherin, What changes in conceptual change, Int. J. Sci. Educ. 20, 1155 (1998).

[59] O. Parnafes, What does fast mean? Understanding the physical world through computational representations, J. Learn. Sci. 16, 415 (2007). 